\title{
e-Health Services in the Context of IoT: The Case of the VICINITY project
}

\author{
Maria Belesioti ${ }^{1}$, Ioannis P. Chochliouros ${ }^{1}$, Stefan Vanya ${ }^{2}$, Viktor Oravec ${ }^{2}$, \\ Natalia Theologou ${ }^{3}$, Maria Koutli ${ }^{3}$, Athanasios Tryferidis ${ }^{3}$ and Dimitrios Tzovaras ${ }^{3}$ \\ ${ }^{1}$ Hellenic Telecommunications Organization (OTE) S.A., \\ 99, Kifissias Avenue, GR 15124, Maroussi, Athens, Greece \\ \{mbelesioti, ichochliouros\}@oteresearch.gr \\ ${ }^{2}$ bAvenir, s.r.o., \\ Jégého 8, 82108 Bratislava, Slovakia \\ \{stefan.vanya, viktor.oravec\}@bavenir.eu \\ ${ }^{3}$ CERTH/ITI - Centre for Research and Technology Hellas/Information Technologies Institute, \\ 6th km Harilaou - Thermi, 57001, Thessaloniki, Greece \\ \{nataliath, mkoutli, thanasic, dimitrios.tzovaras\}@iti.gr

\begin{abstract}
The Internet of Things (IoT) is a new paradigm that combines aspects and technologies coming from different approaches. Ageing population and decreasing financial resources, consist one of the biggest challenges not only in Europe but also worldwide, in terms of healthcare organization complexity. At the same time, there exists an ever-growing demand for ubiquitous healthcare systems to improve human health and well-being. IoT paradigm and wearable IoT devices for home-based or mobile monitoring of vital patients' data can be a secure, reliable and cost-savvy solution to this problem. This paper analyzes the impact of Internet of Things on the design of new eHealth services and solutions in the Context of VICINITY EU-funded project.
\end{abstract}

Keywords: Internet of Things (IoT), eHealth, mobile and portable healthcare devices, interoperability.

\section{$1 \quad$ Introduction}

Recently, there have been significant advances in the field of Internet of Things (IoT) in conjunction to the continuous and constantly increasing demand for support services in the emerging sector of e-health. Aged people with chronic diseases are in need for care and prevention of accidents at 24/7 basis, thus the need to define new models of healthcare that will advance existing systems is of high importance for modern electronic communications market sector. The VICINITY EU-funded project under Grant Agreement (GA) No.688467 [http://vicinity2020.eu/vicinity/] aims to illustrate that the communication models, protocols and technologies promoted under 
the IoT concept have a great potential in the implementation of Internet-based healthcare systems. In addition, VICINITY intends to provide the owners of connected IoT infrastructures with a decentralized interoperability. The concept of "decentralism" is expressed by the fact that the corresponding platform includes neither central operator roles nor central databases to store sensitive data about the involved users. Instead of that, it connects different smart objects into a "social network" called as "virtual neighborhood" where infrastructure owners keep under control their shared devices and data, thanks to web-based operator console called as the "VICINITY neighborhood manager (VNM)". When using the VNM, the user can control which of his/her IoT asset is shared with whom, and to which extent [10]. This VICINITY-related technology aspires to apply in its e-health scenario.

E-health comprises a novel combination of Telemedicine and IoT-based monitoring system and it is considered as one of "the most promising domains in the field of IoT", since it can offer effective and direct healthcare services improving the quality of existing healthcare systems by supporting reliable and efficient solutions. In order to offer and support several e-health solutions, VICINITY will build and demonstrate a device and standard agnostic platform for IoT infrastructures that will offer "Interoperability as-a-Service". This platform will rely upon a decentralised and usercentric approach that offer a complete transparency across vertical domains, while retaining full control of the ownership and distribution of data.

In order to realize such a network, a bridging point, the Gateway API (Application Programming Interface) facilitates an exchange of data between a connected IoT infrastructure and Value-added services. This gateway has an overall knowledge and control over both the sensor network and the data to be transmitted. More specifically, The VICINITY Gateway API utilizes a Communication Node with a set of technologies that makes the data transfer possible even in case when an IoT ecosystem resides behind the Network Address Translation - NAT [10].

\section{VICINITY Concept and Approach}

The ongoing digital evolution has very high impact in the healthcare system, by leading to the rapid growth of many healthcare practices supported by electronic processes and communication known as electronic Health (eHealth) applications [2]. The expected huge number of interconnected devices used in the health sector and the significant amount of data gathered by sensors and smart meters create both technical and business opportunities by introducing new services that will bring tangible benefits to the society [5]. Mobile devices, such as cellular phones, wearables and personal digital assistants (PDAs), are very popular and various IoT networks are being deployed for sensing, measuring, controlling and business process optimization purposes, while various IoT platforms are emerging on the market to manage these networks. Since these infrastructures are mostly acting as "isolated islands" in the global IoT landscape, their inter-connection might bring significant value added (such as an ecosystem running on close-to-zero energy for example). Taking this into consideration, VICINITY aims to present a new approach by exploring new opportunities and addressing new challenges. For this reason, the project aims to create new ecosystems, composed by integrated platforms and smart objects which will be 
supported by network technologies and will make use of the evolving ICT advances.

Owners of connected IoT infrastructures could be also connected to this ecosystem, which is called "virtual neighborhood" and which represents a social network where each one will be able to keep under control their shared devices and data, thanks to a web-based operator console called as the "VICINITY neighborhood manager" (VNM). When using the VNM, each user can control which of his/her IoT asset is shared with whom and to which extent, just like they do in existing social networks and, furthermore, to manage access rules to his/her discovered smart objects through a catalogue appeared in the user's device when open the "VICINITY auto discovery device", through the "VICINITY gateway API" with sample implementations. Once user's IoT infrastructure is connected to the VICINITY platform, the IoT value chains become unlocked, thus opening the way towards seamless interoperability between IoT islands present in the current IoT landscape and this enables the exploitation of independent value-added services, including various cross-domain IoT applications (as discussed in [6]).

Figure 1, as illustrated below, depicts a generalized concept coming from the wider VICINITY approach.

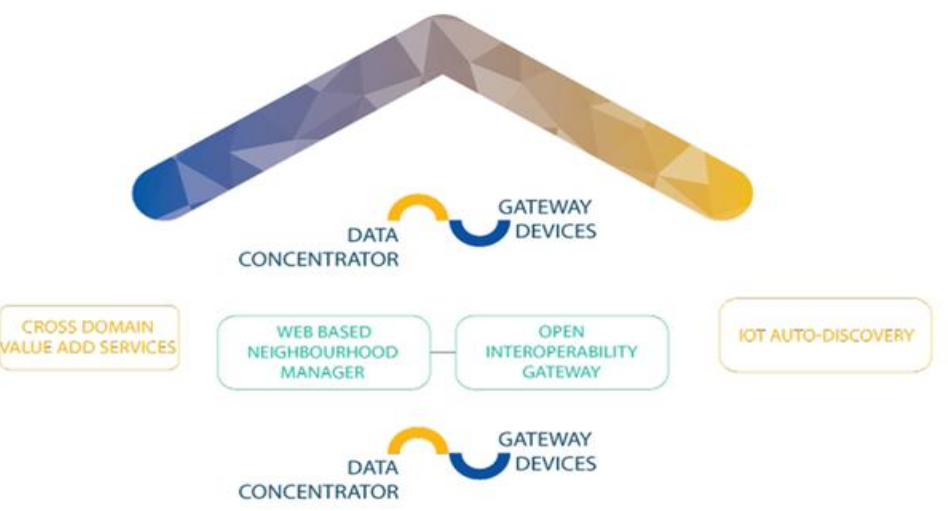

Fig. 1. Visualised VICINITY concept.

The VICINITY quality features focus mainly upon user experience, trust, privacy, security and scalability. VICINITY functions are designed around the user so as to ensure "as best as possible" user experience during installation, configuration, integration of VICINITY components in its infrastructure and usage of the VICINITY interoperability features. The high availability and performance quality measures do enable VICINITY to scale-up and scale-out to "handle" various communication loads within neighborhoods, as introduced by different applications coming from building, energy, transport and health domain requirements.

The VICINITY Cloud components such as the VICINITY Neighborhood manager (providing user interface to the VICINITY Users), Semantic discovery and dynamic configuration agent platform (providing semantic platform) and the VICINITY Communication Server (providing control of communication between integrated infrastructures) shall be deployed as high available software components being to scale 
in/out the VICINITY cloud to current needs to the integrated infrastructures and valueadded services. The distributed infrastructure of the VICINITY context is enhanced by an interoperability approach aiming to provide a standard way to both Discover and Access heterogeneous IoT objects, distributed among sparse IoT infrastructures based on the work being done by the W3C Web of Things (WoT) WG. Therefore, VICINITY shall rely on Thing description (TD) introduced by WoT to describe every IoT object (which can represent either physical or abstract Things) that belong to any integrated IoT infrastructure which, in turn, shall be described as an ecosystem of IoT objects.

The IoT Operator should manage privacy throughout the lifecycle, by using consent to process any sort of private data. These consents should be associated with valueadded services and, additionally, confirmed consents should be part of the value-added service's profile. They should be visible only to the IoT Operator and the Service provider. The second one should also be able to create -or remove- a value-added service group and add -or remove- value-added services from this group and to perform group actions on value-added services (such as changing value-added service access rules). Each value-added service group should have a profile including at least name, avatar, description, value-added service group visibility, accessibility rules, optionally terms and conditions and/or consent to process the private data template. Finally, the device owner should be able to confirm consent so that to process private data by a value-added service -or organization-, which has access to the IoT device and to manage all confirmed consents and revoke them, individually. Furthermore, the device owner should be able to create -or remove- an IoT device group and add -or remove- IoT devices from this group.

The next figure (Fig.2) depicts a schematic view of the essential VICINITY neighborhood concept.

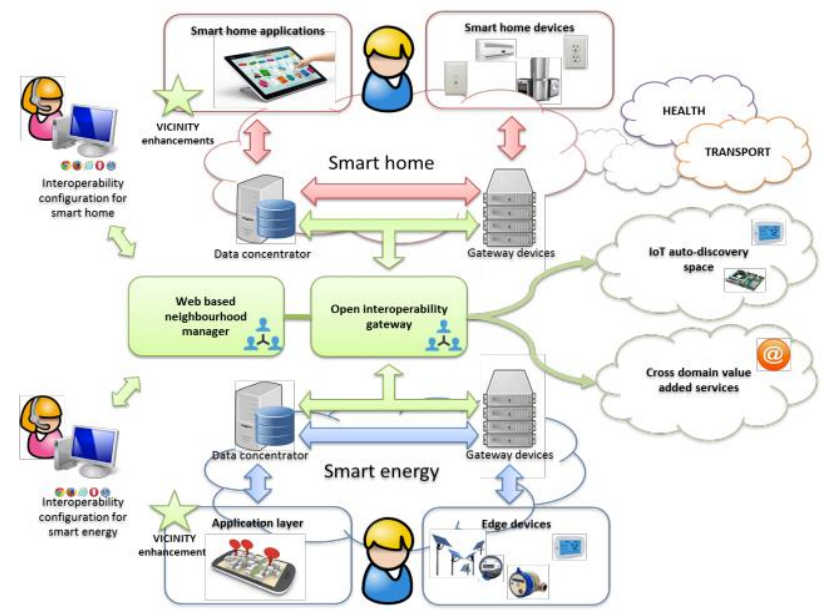

Fig. 2 Schematic view of the VICINITY neighborhood concept [10].

Integrated IoT infrastructures consist of existing IoT assets. The VICINITY Gateway Adapters translate the end-user devices' specific communication protocol into 
VICINITY Interoperability Gateway API calls while, at the same time, the Gateway Adapter will offer a control interface towards the end-user devices by establishing bidirectional communication with the end-user devices. In most of IoT-based patient monitoring systems, especially at smart homes or hospitals, there exists a bridging point (i.e., a gateway) between a sensor network and the Internet, which often just performs basic functions such as translating between the protocols used in the Internet and sensor networks [1]. The VICINITY solution is expected to be based upon an open interoperability gateway, enabling different IoT networks to be connected into large ecosystems. The connections will be organised in a peer-to-per way, building social networks of smart objects (i.e., virtual neighborhoods). The operators of the particular IoT infrastructures can select which other systems they wish to connect to in a similar way as we are selecting friends in social networks, via a web-based neighborhood manager where they can configure the scope of the cooperation with other systems (e.g. to decide which data are visible and which controls are applicable for a particular partner entity). Facilitating the integration of smart objects built on widely adopted standards the platform will be equipped with semantic discovery and dynamic configuration features automatically integrating such assets to VICINITY. At this point, it should be mentioned that several potential barriers could occur, such as:

- Lack of IoT protocol interoperability (systems are often vendor locked by design);

- Interconnected smart objects of different owners require data sharing that raises serious privacy issues;

- IoT component vendors might be reluctant to share interface specifications (Intellectual Property problem), and;

- Large-scale integration imposes rules that are disadvantageous for particular participants.

All these issues are expected to be assessed, in a proper way, within the VICINITY framework.

\section{VICINITY e-Health Scenario}

Digital technologies such as $4 \mathrm{G}$ and the upcoming 5G mobile network, artificial intelligence and IoT-based systems provide new opportunities to transform the way of healthcare services provision. In January 2014, in a research held in the United States, revealed that $85 \%$ of the adult population owns a mobile phone, and nearly 46 million smartphone owners used health or fitness applications (apps) [8]. At the same time, another research, this time on behalf of industry, has reported a $40 \%$ increase among health care professionals such as doctors, who use electronic tools for patient communications, and more than $20 \%$ of physicians now use mobile technologies for remote patient monitoring [9]. These figures create a potential augmentation in distance care practices and VICINITY has already recognized this.

In Europe, eHealth systems are developing despite the existence of many different healthcare systems. According to the European Commission, e-health comprises the following four interrelated categories of applications [6]:

(i) Clinical information systems;

(ii) telemedicine and home care, personalized health systems and services for remote patient monitoring, teleconsultation, telecare, telemedicine and tele- 
radiology;

(iii) integrated regional/national health information networks, distributed electronic health record systems and associated services such as e-prescriptions or ereferrals, and;

(iv) secondary usage of non-clinical systems (such as specialized systems for researchers, or support systems such as billing systems.

E-health still receives a great deal of attention at the EU level, and the Commission has invested in several research programs related to this area [7]. The VICINITY ehealth scenario aims to transform healthcare delivery for elderly patients with long terms needs (such as people with dementia and obesity) and to provide a scalable and holistic approach in healthcare systems. Moreover, it shall be deployed where the term "virtual neighborhood" is referred to supporting communication of personal health status data to selected participants of the network such as family members, for example. The novelty of VICINITY project in the e-health domain is due to the fact that participants can decide with whom they wish to cooperate and to which extent. Thus, each participant can decide on the conditions for himself in his own maximal favor.

The VICINITY ehealth scenario is divided to two sub-scenarios: The first one is called ehealth \& assisted living and its main purpose is to demonstrate, through constant remote monitoring, how sensors, actuators and integrated communication devices installed at home can provide assisted living to elderly people and people with long-terms needs, as previously mentioned. The second scenario is about fitness and preventive medicine with wearable IoT and aims mainly at middle-aged persons, who need to promote their health as a method of diseases preventions. Unique identification of the user is of high importance in this use case. VICINITY platform repeatedly associates the entities within the system with an individual name, code, symbol, or number and offering interaction with the entities, or tracing and controlling their activities. This distinction is the key enabler for interoperability and global services across heterogeneous IoT systems and it is important for entities to be uniquely identifiable so that IoT systems can monitor and communicate with specific entities.

Fig.3 illustrates as schematic view of the VICINITY e-health scenario in Greece, as proposed by the national involved partners. The entire approach is based upon the broader VICINITY context.

The sensors and wearables will be in direct communication with a call center manned with specialized staff and maybe doctors or alternatively with their relatives. The real-time combination of information, provided by the sensors, creates a unique user profile and, therefore, any deviation from this is characterized as "abnormal behavior" thus triggering an alarm. The target of the ehealth scenario is to assist both elderly and middle-aged people to support their everyday life by offering them a secure and independent way of living. This VICINITY service has all the potential to be a new promising vehicle able to enhance the limited abilities of the healthcare system by assisting patients with chronic disease management and monitoring and by tracking their everyday activities so as to promote a healthier lifestyle. Of course, despite these potential benefits, there several issues and barriers and limitations that should overcome and many quality measures that should be addressed ([3], [4], [12], [13]). 


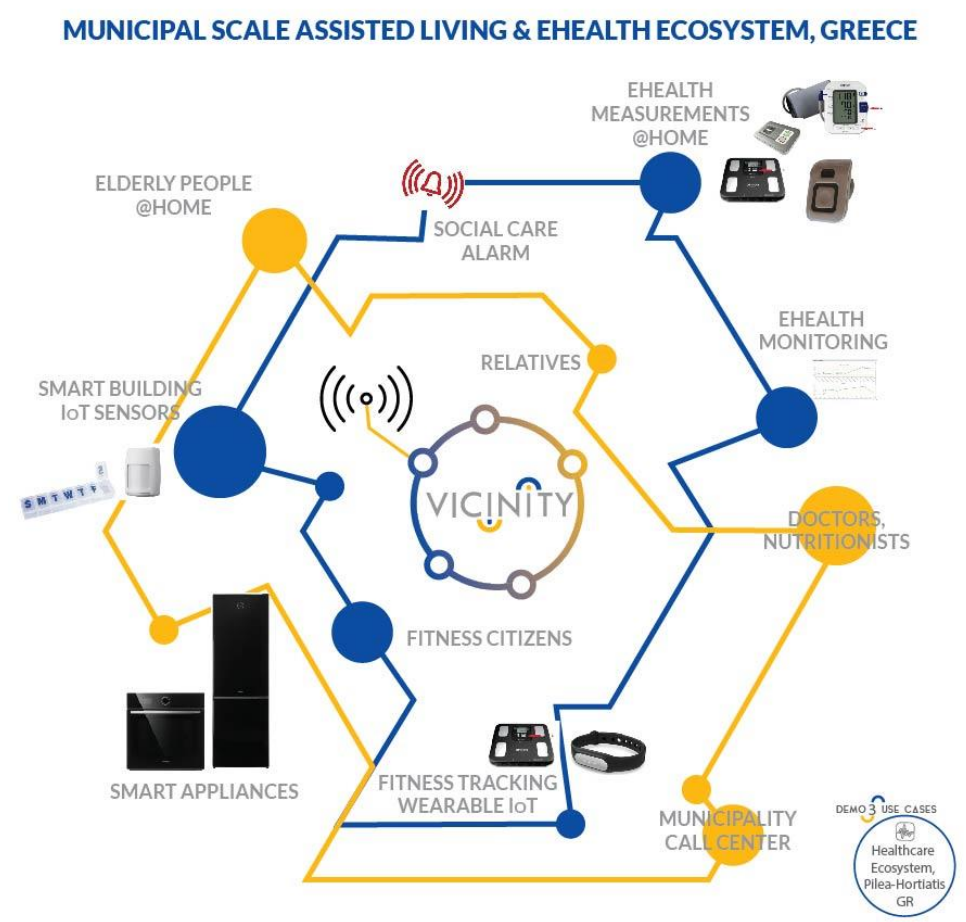

Fig.3. Schematic view of the VICINITY e-Health scenario in Greece [11].

\section{Conclusion}

The importance of prevention and monitoring in the health sector becomes clear when the combined effects of the limited capacity of the existing healthcare system to support the increasing demands of the aging population, and the continues increase of need for disease prevention and healthier way of living expand. Using information technology to monitor patient's care and if need be to assist them, may have a positive impact especially in developing countries?

The evolution of IoT has created a variety of application in several domains, including healthcare. EHealth could transform healthcare delivery by offering the potential to improve the quality, accountability, and cost-effectiveness of healthcare services 0 . VICINITY project through its "virtual neighborhood" and its applications aims to redesign modern healthcare services with promising technological, economic, and social prospects. The proposed services can have positive results like improved everyday life with increased quality.

Acknowledgments. This work has been performed in the scope of the VICINITY European Research Project and has been supported by the Commission of the European Communities (H2020, Grant Agreement No.688467). This paper reflects 
only the authors' views and the Commission is not liable for any use that may be made of the information contained therein.

\section{References}

1. Rahmani, A.M., et al.: Smart e-health Gateway: Bringing intelligence to Internet-of-Things based ubiquitous healthcare systems. In Proceedings of the $201512^{\text {th }}$ Annual IEEE Consumer Communications and Networking Conference (CCNC), Las Vegas, NV, pp.826$834(2015)$

2. An Open NCP-based Secure eHealth Data Exchange System. Available at: https://www.researchgate.net/publication/322775597_KONFIDO_An_OpenNCP-

based_Secure eHealth_Data_Exchange_System [accessed Feb.01, 2018]

3. Clarke, G., and Yarborough, B.J.: Evaluating the promise of health IT to enhance/expand the reach of mental health services. General Hospital Psychiatry, 35(4), 339-344 (2014)

4. Chang, B.L., Bakken, S., Brown, S.S., Houston T.K., et al.: Bridging the digital divide: reaching vulnerable populations. Journal of American Medical Informatics Association, 11(6), 448-457 (2004)

5. Borgia, E.: The Internet of Things vision: Key features, applications and open issues. Computer Communications 54(1), 1-31 (2014)

6. eHealth Taskforce: Accelerating the Development of the eHealth Market in Europe. eHealth Taskforce Report (2007), p.10

7. European Commission: eHealth portfolio of projects. European Commission Information Society and Media, Brussels (2007)

http://ec.europa.eu/information_society/activities/health/docs/publications/fp6upd2007/fp6in tro1.pdf

8. Nielsen: Hacking health: how consumers use smartphones and wearable tech to track their health. Nielsen (March 2015). Available from:

www.nielsen.com/us/en/insights/news/2014/hacking-health-how-consumers-usesmartphones-and-wearable-tech-to-track-their-health.html.

9. Terry, K.: Physicians warm to digital communications with patients. (2014). Available from: www.medscape.com/viewarticle/826596.

10. VICINITY Project, GA No.688467: Open virtual neighborhood network to connect IoT Infrastructures and smart objects. [http://vicinity2020.eu/vicinity/]

11. VICINITY Project: Pilea-Hortiatis (GR) eHealth \& Assisted Living. http://vicinity2020.eu/vicinity/content/pilea-hortiatis-gr-\%E2\%80\%93-ehealth-assistedliving

12. Walji, M., Sagaram, S., Sagaram, D., Meric-Bernstam, F., Johnson, C., Mirza Nadeem, Q., Bernstam Elmer V.: Efficacy of quality criteria to identify potentially harmful information: a cross-sectional survey of complementary and alternative medicine web sites. Journal of Medical Internet Research, 29(6(2):e21, pp.87-100 (2004)

13. Sagaram, S., Walji, M., Meric-Bernstam, F., Johnson, C., and Bernstam, E.: Inter-observer agreement for quality measures applied to online health information. Studies in Health Technology and Informatics, 107(Pt.2), pp.1308-1312 (2004) 